\title{
Cyclic and dihedral 1-factorizations of multipartite graphs
}

\author{
Mathieu Bogaerts * Giuseppe Mazzuoccolo ${ }^{\dagger}$ \\ Submitted: Nov 18, 2010; Accepted: Sep 1, 2011; Published: Sep 9, 2011 \\ Mathematics Subject Classification: 05C25; $05 \mathrm{C} 70$
}

\begin{abstract}
An automorphism group $G$ of a 1-factorization of the complete multipartite graph $K_{m \times n}$ consists of permutations of the vertices of the graph mapping factors to factors. In this paper, we give a complete answer to the existence problem of a 1-factorization of $K_{m \times n}$ admitting a cyclic or dihedral group acting sharply transitively on the vertices of the graph.
\end{abstract}

\section{Introduction}

In this paper, we consider 1-factorizations of complete multipartite graphs $K_{m \times n}$ (i.e. with $m$ parts consisting of $n$ vertices). In order to avoid confusion with the complete graph $K_{m}=K_{m \times 1}$, it is from now assumed that each part of $K_{m \times n}$ has at least two vertices (i.e. $n \geq 2$ ). An $r$-factor of a graph $\Gamma$ is a spanning subgraph with all vertices of degree $r$. For $r=1$, the concept of 1-factor coincide with the definition of a perfect matching. An $r$-factorization of a graph $\Gamma$ is a partition of $E(\Gamma)$ into disjoint $r$-factors.

A group of permutations of the vertices of $\Gamma$ mapping factors to factors is called an automorphism group of the $r$-factorization. If the action of an automorphism group is sharply transitive on the vertices, the factorization is said to be sharply transitive.

In [7], G. Rinaldi and the second author consider the following problem:

Problem 1. Given a finite group $G$ of even order, which graphs $\Gamma$ have a 1-factorization admitting $G$ as an automorphism group with a sharply transitive action on the vertex set?

The problem above is the natural generalization of the largely studied case in which we set $\Gamma$ to be the complete graph. The benchmark on this topic is the following theorem of Hartman and Rosa [4] from 1985:

*Faculté des Sciences Appliquées, Université Libre de Bruxelles, CP 165/11 avenue Roosevelt 50, Bruxelles Belgium. email: mbogaert@ulb.ac.be

${ }^{\dagger}$ Dipartimento di Matematica, Università di Modena e Reggio Emilia, via Campi 213/B, I-41100 Modena, Italy. email: giuseppe.mazzuoccolo@unimore.it 
Theorem 1. A complete graph $K_{n}$ admits a 1-factorization with a cyclic automorphism group acting sharply transitively on the vertices if and only if $n$ is even and $n \neq 2^{t}, t \geq 3$.

Later on, other specified classes of groups have been considered by various authors; among others we would like to recall the generalizations of Theorem 1 to the entire classes of finite abelian groups (Buratti, [3]) and finitely generated abelian groups (Bonvicini and Mazzuoccolo, [2]). Even if Problem 1 remains still open if $\Gamma$ is the complete graph, it is interesting to consider other possible choices for the graph $\Gamma$ : for instance, the main result of [7] states the existence and non-existence of a 1-factorization of the complete multipartite graph admitting a cyclic automorphism group acting sharply transitively on the vertex-set (a cyclic 1-factorization from now on).

Theorem 2. [7] A cyclic 1-factorization of $K_{m \times n}$ :

- does not exist if $m \equiv 3 \bmod 4, n=2 d$ where $d$ is odd

- does not exist if $m=2^{v} d, n=2 d^{\prime}$ where $d$ and $d^{\prime}$ are odd and $v \geq 2$

- exists if $m=2 d, n=2 d^{\prime}$ where $d$ and $d^{\prime}$ are odd.

- exists if $m=2^{v} d, n=2^{u} d^{\prime}$ where $d$ and $d^{\prime}$ are odd and $u>1$.

- exists if $m=2^{v} d, n=d^{\prime}$ where $d$ and $d^{\prime}$ are odd and $v \geq 1$.

The following theorem is the first main result of this paper and completes all the open cases of Theorem 2 (i.e. $m \equiv 1 \bmod 4, n=2 d$ with $d$ odd). It is obtained by combining Theorem 8 and Theorem 9 .

Theorem 3. A cyclic 1-factorization of $K_{m \times n}$ :

- does not exist if $m=p^{v}, n=2$, where $p$ is prime such that $p \equiv 1 \bmod 4$ and $v \geq 1$,

- exists if $n=2, m \equiv 1 \bmod 4$ and $m$ is not a prime power,

- exists if $m \equiv 1 \bmod 4$ and $n=2 d$ where $d>1$ is odd.

The material involved in the constructions is presented in section 2. Section 3.1 states the existence and non-existence result for $n=2$, while the construction for $n=2 d$ ( $d>1$ odd) is presented in section 3.2 .

In [1], the case of 1-factorizations of the complete graph admitting a dihedral group acting sharply transitively on the vertices (dihedral 1-factorizations in what follows) is settled; in section 4, two "doubling" constructions and three appropriate constructions are described in order to prove an equivalent result for complete multipartite graphs. Namely, we prove that a dihedral 1-factorization of $K_{m \times n}$ exists for all $m, n$ with $m n$ even (see Theorem 13). 


\section{Preliminaries}

It is proved in [7] that the existence of a 1-factorization of $K_{m \times n}$ having an automorphism group $G$ acting sharply transitively on the vertices is equivalent to the existence of a particular starter, a very slight generalization of the concept of a starter introduced by Buratti in [3] for the complete graph. First, the graph $\Gamma=K_{m \times n}$ has to be considered as the Cayley graph $\operatorname{Cay}(G, \Omega)$ with $\Omega=G-H$ where $G$ is a group of order $m n$ and $H$ is a subgroup of order $n$ of $G$. The vertices of $K_{m \times n}$ are identified with elements of $G$, and edges are (unordered) pairs of elements $\left[g_{1}, g_{2}\right], g_{i} \in G$ such that the "difference" $g_{1} g_{2}^{-1}$ belongs to $\Omega$.

Edges in $\Gamma$ don't necessarily have the same kind of orbits under the action of $G$. The set $\Omega$ can be partitioned as $\Omega_{1} \cup \Omega_{2} \cup \Omega_{2}^{-1}$ where $\Omega_{1}$ contains all involutions of $\Omega$, and for any $g \in \Omega_{2}, g^{-1} \in \Omega_{2}^{-1}$. The edge set of $\Gamma$ can be described as $\left(\cup_{g \in \Omega_{1}} \operatorname{Orb}_{G}\left(\left[1_{G}, g\right]\right)\right) \cup$ $\left(\cup_{h \in \Omega_{2}} \operatorname{Orb}_{G}\left(\left[1_{G}, h\right]\right)\right)$, where $1_{G}$ denotes the identity element of $G$. For any $g \in \Omega_{1}$, $\operatorname{Orb}_{G}\left(\left[1_{G}, g\right]\right)$ has $\frac{m n}{2}$ elements and forms a 1-factor of $\Gamma$. Because there are only $\frac{m n}{2}$ elements in each orbit, edges of this kind are called short edges.

On the other hand, for $g \in \Omega_{2}, \operatorname{Orb}_{G}\left(\left[1_{G}, g\right]\right)$ has $m n$ elements and is a union of cycles of $\Gamma$. These cycles can be described as $\left(x, g x, g^{2} x, \ldots, g^{k-1} x\right)$ where $k$ is the order of $g$ and $x$ is a representative of one right coset of $\langle g\rangle$ in $G$. The edges in these orbits are called long edges.

Now we define two mappings $\partial, \phi$. The first one maps an edge to the differences of its vertices, and the second one maps an edge to its vertices. Both give one element if the edge is short, and 2 if the edge is long.

$$
\begin{gathered}
\partial([x, y])= \begin{cases}\left\{x y^{-1}, y x^{-1}\right\} & \text { if }[x, y] \text { is long } \\
\left\{x y^{-1}\right\} & \text { if }[x, y] \text { is short }\end{cases} \\
\phi([x, y])= \begin{cases}\{x, y\} & \text { if }[x, y] \text { is long } \\
\{x\} & \text { if }[x, y] \text { is short }\end{cases}
\end{gathered}
$$

Then for a set $S$, we define $\partial(S)=\cup_{e \in S} \partial(e)$ and $\phi(S)=\cup_{e \in S} \phi(e)$.

Now we give the definition of a starter for the pair $(G, \Omega)$ : it is a set $\Sigma=\left\{S_{1}, \ldots, S_{k}\right\}$ of subsets of the edges $E(\Gamma)$, with $k$ subgroups $H_{1}, \ldots, H_{k}$ of $G$ such that:

- the union of the differences $\partial S_{1} \cup \cdots \cup \partial S_{k}$ is $\Omega$, but without repetition (every element appears exactly once).

- for each $S_{i}, \phi\left(S_{i}\right)$ contains exactly one representative of each right coset of $H_{i}$ in $G$.

- for any $H_{i}$ and any short edge $[x, y] \in S_{i}, H_{i}$ contains the involution $x^{-1} y=y^{-1} x$.

Theorem 4. [7] There exists a starter for $(G, \Omega)$ if and only if there exists a 1-factorization of $\Gamma=\operatorname{Cay}(G, \Omega)$ admitting $G$ as automorphism group with a sharply transitive action on the vertices. 


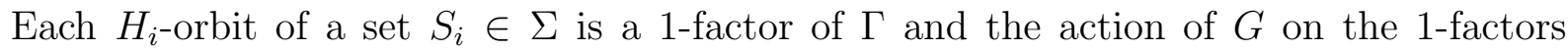
covers all edges of $\Gamma$ exactly once. By construction, the factorization is preserved under the action of $G$.

In the next sections we will largely make use of the fact that the construction of a starter can be simplified by the existence of a subgroup $A$ of $G$ of index 2 , as mentioned in [7]:

Proposition 5. [7] Let $G$ be a finite group possessing a subgroup $A$ of index 2 and let $\Sigma^{\prime}=$ $\left\{S_{1}, \ldots, S_{t}\right\}$ be a set of subsets of $E(\Gamma)$ together with subgroups $H_{1}, \ldots, H_{t}$ which satisfy the second and third conditions of the definition of a starter. If $A \cap \Omega \subset \partial S_{1} \cup \cdots \cup \partial S_{t} \subset \Omega$ and it does not contain repeated elements, then $\Sigma^{\prime}$ can be completed to a starter for the $\operatorname{pair}(G, \Omega)$.

\section{Cyclic 1-factorization of $K_{m \times 2 d}, m \equiv 1 \bmod 4$}

In all this section, the group $G$ acting on the vertices of the graph is cyclic and is supposed to be written additively.

\subsection{The case $d=1$}

The graph $K_{m \times 2}$ can be obtained by deletion of a 1-factor $F$ of the complete graph $K_{2 m}$, hence the existence of a cyclic 1-factorization of $K_{m \times 2}$ is exactly equivalent to the existence of a cyclic 1-factorization of $K_{2 m}$ with an invariant 1-factor $F$. Korchmáros, in [6], conjectured that no sharply-transitive 1-factorization with an invariant 1-factor exists if $m \equiv 1 \bmod 4$, but Rinaldi in [8] provided an example of a cyclic 1-factorization of $K_{21 \times 2}$.

A relevant progress on this problem is obtained by the main result of [5]: Theorem 1.1 of [5] states the existence of a 2-factorization of $K_{2 m}-F$, the complete graph minus a 1 -factor $F$, preserved by a cyclic group of order $2 m$ acting sharply transitively on the vertices for all $m \equiv 1 \bmod 4$ and $m \neq p^{v}$ with $p$ prime. In this construction, each 2-factor of $K_{m \times 2}$ is an hamiltonian cycle on an even number of vertices, then it can be decomposed in two disjoint 1-factors. It is an easy check that the cyclic group of order $2 m$ preserving the 2-factorization is also an automorphism group of the obtained 1-factorization, and so the following proposition holds.

Proposition 6. A cyclic 1-factorization of $K_{m \times 2}$ exists for each $m \equiv 1 \bmod 4$ and $m \neq p^{v}$ with $p$ prime.

The non-existence of a cyclic 1 -factorization of $K_{m \times 2}$ with $m \equiv 3 \bmod 4$ is contained in Theorem 2, whereas Rinaldi in [8] proves the non-existence of a cyclic 1-factorization of $K_{m \times 2}$, for $m \equiv 1 \bmod 4$ and $m$ prime. In the following proposition we generalize that result:

Proposition 7. A 1-factorization of $K_{m \times 2}$ with $m=p^{v}$ with $p$ prime, $v \geq 1$ cannot admit a cyclic automorphism group acting sharply transitively on the vertex-set. 
Proof. Suppose the existence of a starter $\Sigma=\left\{S_{1}, \ldots, S_{k}\right\}$ with subgroups $H_{1}, \ldots$, $H_{k}$, respectively.

Note that if $G$ is the cyclic group of order $2 m=2 p^{v}$ where $p$ is an odd prime and $v \geq 1$, then the only subset $\Omega$ that permits the construction of $K_{m \times 2}$ is $G \backslash\left\langle p^{v}>\right.$.In this case, since the unique involution of $G$ does not belong to $\Omega$, all edges are long, and for all $i, \phi\left(S_{i}\right)$ has an even number of elements.

By definition, in order to form a transversal for $H_{i}$, the subset $\phi\left(S_{i}\right)$ must contain $\frac{|G|}{\left|H_{i}\right|}$ elements, and since this number is even, all $H_{i}$ involved in the construction of the starter must have odd order. The possible orders for $H_{i}$ are $p, p^{2} \ldots p^{v}$ and so each $H_{i}$ must be generated by one of the elements $2 p^{v-1}, \ldots, 2 p^{2}, 2 p, 2$, respectively. In each case, the element $2 p^{v-1}$ belongs to $H_{i}$, and cannot be covered in $\partial\left(S_{i}\right)$. This proves that such a starter $\Sigma$ cannot exist and therefore, the non-existence of the 1-factorization.

Proposition 6, together with Proposition 7, gives a complete description of the existence spectrum for a cyclic 1-factorization of $K_{m \times 2}$. We briefly summarize it in the following theorem:

Theorem 8. A cyclic 1-factorization of $K_{m \times 2}$, with $m>1$ odd, exists if and only if $m \equiv 1 \bmod 4$ and $m \neq p^{v}$ with $p$ prime.

\subsection{The case $d>1$}

Differently from what happens in the case $d=1$, where both existence and non-existence results have appeared, we are able to furnish a cyclic 1-factorization for each odd value of $d>1$ and for each $m \equiv 1 \bmod 4$.

Theorem 9. For all $m \equiv 1 \bmod 4, d \neq 1$ odd, there is a 1-factorization of $K_{m \times 2 d}$ admitting the cyclic group $\mathbb{Z}_{2 d m}$ as automorphism group acting sharply transitively on the vertices .

Proof. Let define the $\frac{d+1}{2}$ sets $S_{k}$ with corresponding subgroup $H_{k}=<2 m>$ of order d. For $1 \leq k \leq \frac{d-1}{2}$,

$$
S_{k}=\left\{[2 t, 2 k m-2-2 t],[3+2 t, 2 k m-1-2 t]: t=0, \ldots, \frac{m-3}{2}\right\} \cup\{[1,2 k m-m-1]\}
$$

The set $S_{\frac{d+1}{2}}$ is defined as:

$$
S_{\frac{d+1}{2}}=S_{\frac{d+1}{2}}^{1} \cup S_{\frac{d+1}{2}}^{2} \cup\left\{\left[m-1-\frac{m-3}{2}, m-1\right]\right\}
$$

where $S_{\frac{d+1}{2}}^{1}$ and $S_{\frac{d+1}{2}}^{2}$ are:

$$
\begin{gathered}
S_{\frac{d+1}{2}}^{1}=\left\{[m-2 t, d m+2+2 t],[m-3-2 t, d m+1+2 t]: t=0, \ldots, \frac{m-5}{4}\right\} \\
S_{\frac{d+1}{2}}^{2}=\left\{[t,(d+1) m-1-t]: t=0, \ldots, \frac{m-3}{2}\right\} .
\end{gathered}
$$


Note that all even integers of the form $\{ \pm((d-1) m+2), \pm((d-1) m+4), \ldots, \pm(m d-1)\}$ are not covered by $\bigcup_{k=1}^{\frac{d-1}{2}} \partial\left(S_{k}\right)$ but are covered in $\partial\left(S_{\frac{d+1}{2}}^{1}\right)$.

It can be directly checked that the set $\Sigma^{\prime}=\left\{S_{1}, \ldots, S_{\frac{d+1}{2}}\right\}$ together with subgroups $H_{1}, \ldots, H_{\frac{d+1}{2}}$ satisfy the second and third conditions of the definition of a starter. The union $\partial S_{1} \cup \cdots \cup \partial S_{\frac{d+1}{2}}$ is contained in $\Omega$ and contains all elements of $A \cap \Omega$ without repetition, where $A=<2>$ is the subgroup of index 2 of $\mathbb{Z}_{2 d m}$. Proposition 5 can be applied, and $\Sigma^{\prime}$ can be completed to a starter for $(G, \Omega)$.

\section{Dihedral factorization of $K_{m \times n}$}

In all this section, the dihedral group $G=D_{k}$ is considered as generated by an element $a$ of order $k$ and an involution $b$ such that $b a=a^{-1} b$.

For all $n$, a 1-factorization of $K_{2 \times n}$ having $G=D_{n}$ (of order $2 n$ ) as sharply transitive automorphism group exists. It is an immediate consequence of the result of Mazzuoccolo and Rinaldi [7], since they proved that if $G$ has a subgroup of index 2, then such factorization exists.

Recall that the graph $K_{m \times 2}$ can be seen as the complete graph $K_{2 m}$ minus a 1-factor. For each value of $m$, the constructions in [1] give a 1-factorization of $K_{2 m}$ preserved by a dihedral group acting sharply transitively on the vertex-set and fixing at least one 1factor. Removing this factor of the factorization of $K_{2 m}$ provides a 1-factorization of $K_{m \times 2}$, preserved by the same dihedral group, acting sharply transitively on the vertices.

Proposition 10 (First doubling construction). If there exists a cyclic 1-factorization of $K_{m \times n}$ then there exists a dihedral factorization of $K_{m \times 2 n}$.

Proof. Let suppose that the cyclic 1-factorization of $K_{m \times n}$ is constructed with $H^{\prime}=<$ $a^{m}>$. The vertices of $K_{m \times n}$ are identified with the powers of $a$ in $K_{m \times 2 n}$. For each ordered pair $\left(S_{i}^{\prime}, H_{i}^{\prime}\right)$ of the starter in $K_{m \times n}$, we construct the set $S_{i}=S_{i}^{\prime}$ (as subset of $G=D_{m n}=<a, b>$ ) with group $H_{i}=<H_{i}^{\prime}, b>($ as a subgroup of $G)$.

The edges not covered are all short edges of $K_{m \times n}$ with difference $a^{j} b$ for any $j$ not divisible by $m$. A starter of $(G, \Omega)$ is obtained with Proposition 5 , adding sets $\left\{\left[1_{G}, a^{j} b\right]\right\}$ with group $D_{m n}$ to the others sets $S_{i}$ 's.

Proposition 11 (Second doubling construction). If there exists a cyclic 1-factorization of $K_{m \times n}$ then there exists a dihedral factorization of $K_{2 m \times n}$.

Proof. The subgroup $H^{\prime}$ used to construct the cyclic factorization of $K_{m \times n}$ is identified with the subgroup $H$ (generated by some power of $a$ ) of $G=D_{m n}$. Taking $\Omega$ as $D_{m n}-H$, the Cayley graph $\operatorname{Cay}(G, \Omega)$ is $K_{2 m \times n}$.

We construct a dihedral factorization of $K_{2 m \times n}$ with the first $S_{i}$ 's as in the factorization of $K_{m \times n}$ and the first new $H_{i}$ 's as: $H_{i}=<H_{i}^{\prime}, b>$. Cosets of $H_{i}$ in $G$ are identified with the cosets of $H_{i}^{\prime}$ in $\mathbb{Z}_{m n}$, and the differences sets $\partial\left(S_{i}\right)$ cover all powers of $a$ in $\Omega=G-H$. 
The starter is obtained by application of Proposition 5: the new $S_{i}$ 's are chosen as $S_{i}=\left\{\left[1_{G}, b a^{j}\right]\right\}$ (one short edge), $j=0, \ldots, m n-1$, together with group $H_{i}=G$. Differences cover all elements written as $a^{j} b$ in $\Omega$, including $b$.

Proposition 12. There exists a 1-factorization of $K_{m \times n}$ admitting a dihedral automorphism group acting sharply transitively on the vertices for:

A. $m=d, n=4 d^{\prime}, d$ and $d^{\prime}$ both odd.

B. $m=d, n=2 d^{\prime}, d$ and $d^{\prime}$ both odd.

C. $m=2 d, n=d^{\prime}, d$ and $d^{\prime}$ both odd.

\section{Proof.}

Construction for case A: The automorphism group considered is $G=D_{2 d d^{\prime}}(d$ and $d^{\prime}$ odd) generated by $a$ and $b$ with $a^{2 d d^{\prime}}=b^{2}=1_{G}$ and $a^{-1}=b a b$. The graph $K_{d \times 4 d^{\prime}}$ is obtained as $\operatorname{Cay}(G, \Omega)$ with $\Omega=G-H$ and $H=<a^{d}, b>$. As required, the subgroup $H$ has order $4 d^{\prime}$. The set $\Omega$ is decomposed as $\Omega_{1} \cup \Omega_{2} \cup \Omega_{2}^{-1}$. The elements of $\Omega_{1}$ can be written as $a^{k} b$ with $k \neq \equiv 0 \bmod d$. The set $\Omega_{2} \cup \Omega_{2}^{-1}$ consists only of $2(d-1) d^{\prime}$ powers of $a$ of the form $a^{k}$ with $k \not \equiv 0 \bmod d$.

The starter is composed of the sets $S_{i}$, constructed as follow. The $d^{\prime}$ first $S_{i}$ are all of the form:

$$
S_{i}=\left(\cup_{j}\left\{\left[a^{j}, a^{-j}\right]\right\}\right) \cup\left\{\left[1_{G}, a^{i d+1} b\right]\right\}
$$

More precisely, the sets $S_{i}, i=0, \ldots, d^{\prime}-1$, are defined as:

$$
S_{i}= \begin{cases}\left(\bigcup_{k=1}^{\frac{d-1}{2}}\left\{\left[a^{i d+k}, a^{-i d-k}\right]\right\}\right) \cup\left\{\left[1_{G}, a^{i d+1} b\right]\right\} & \text { if } i \text { is even } \\ \left(\bigcup_{k=\frac{d+1}{2}}^{d-1}\left\{\left[a^{i d+k}, a^{-i d-k}\right]\right\}\right) \cup\left\{\left[1_{G}, a^{i d+1} b\right]\right\} & \text { if } i \text { is odd }\end{cases}
$$

For each of these $S_{i}$, the corresponding $H_{i}$ is $\left\langle a^{d}, a b\right\rangle$. Each coset of $H_{i}$ is represented in $\phi\left(S_{i}\right)$ by a power of $a$ or the identity. The edges $\left[1_{G}, a^{i d+1} b\right]$ are short (for all $k, a^{k} b$ is an involution), and the involution associated to this edge is in $H_{i}$.

The union $\partial\left(S_{0}\right) \cup \ldots \partial\left(S_{d^{\prime}-1}\right)$ covers all $(d-1) d^{\prime}$ even powers of $a$ that belong to $\Omega_{2} \cup \Omega_{2}^{-1}$ and the $d^{\prime}$ involutions $a^{i d+1} b\left(i=0 \ldots d^{\prime}-1\right)$. The next $\frac{(d-1) d^{\prime}}{2}$ sets $S_{i}$ 's will cover the odd powers of $a$ in $\Omega_{2}$ :

$$
S_{i}=\left\{\left[1_{G}, a^{2 k-1}\right]\right\} \quad \text { with } 2 k-1 \not \equiv 0 \quad \bmod d, \quad 2 k-1 \leq d d^{\prime}-2 \quad \text { and } H_{i}=<a^{2}, b>
$$

Each $H_{i}$ is a subgroup of index 2 of $G$, the elements $1_{G}$ and $a^{2 k-1}$ are representatives of the two cosets of $H_{i}$. The differences $\partial\left(S_{i}\right)$ cover the odd powers of $a$ that appear in $\Omega_{2}^{-1}$. For now, the uncovered edges are all involutions. For each involution we form a set $S_{i}=\left\{\left[1_{G}, a^{k} b\right]\right\}$ and take the corresponding $H_{i}$ to be $G$ itself. There are $2 d d^{\prime}-d^{\prime}$ such 
involutions in $\Omega_{2}$. Note that $d^{\prime}$ of them (of form $a^{i d+1} b$ ) are already covered by the first $S_{i}$ 's. Now, all elements in $\Omega$ are covered exactly once by $\bigcup \partial\left(S_{i}\right)$. The short edges that appears in the $S_{i}$ always have an associated involution contained in $H_{i}$.

Construction for case B: The group $G$ is $D_{d d^{\prime}}\left(d\right.$ and $d^{\prime}$ odd), generated by a and $b$, with $a^{d d^{\prime}}=b^{2}=1_{G}$ and $a^{-1}=b a b$. The subgroup $H$ is $\left\langle a^{d}, b\right\rangle$, of order $2 d^{\prime}$. The set $\Omega=G-H$ is partitioned as $\Omega=\Omega_{1} \cup \Omega_{2} \cup \Omega_{2}^{-1}$. The elements in $\Omega_{1}$ are $(d-1) d^{\prime}$ involutions that can be written as $a^{k} b, k \neq 0 \bmod d$. One way to define $\Omega_{2}$ is the following: $\Omega_{2}=\left\{a^{2 j}: j=1, \ldots, \frac{d d^{\prime}-1}{2}, j \not \equiv 0 \bmod d\right\}$. With this definition, $\Omega_{2}^{-1}$ is composed of the odd powers of $a$. The $d^{\prime}$ first $S_{i}$ 's $\left(i=0, \ldots d^{\prime}-1\right)$ are formally defined as in the construction for case A (but $a$ and $b$ now belong to $D_{d d^{\prime}}$ ) and the corresponding $H_{i}$ 's are equal to $\left\langle a^{d}, a b>\right.$ as in case $\mathrm{A}$.

All elements of $\Omega-\Omega_{1}$ are covered by the union of the difference sets $\partial\left(S_{0}\right) \cup \cdots \cup$ $\partial\left(S_{d^{\prime}-1}\right)$. Some involutions are also covered, and the rest of $\Omega_{1}$ will be covered by the differences sets of the remaining $S_{i}$ 's, defined as: $S_{i}=\left\{\left[1_{G}, a^{k} b\right]\right\}$ with $k \not \equiv 0 \bmod d$, if $a^{k} b$ does not appear in the previous $S_{i}$ 's. The corresponding $H_{i}$ 's are all equal to $G$.

Construction for case C: As in case B, we take $G=D_{d d^{\prime}}$, but $H$ is now equal to $\left\langle a^{d}\right\rangle$ (of order $d^{\prime}$ ). Elements of $\Omega_{2} \cup \Omega_{2}^{-1}$ are exactly the same as in case B. The first $d^{\prime}$ sets can be constructed as in case $\mathrm{B}$, but it is also possible to define it in an easier way:

$$
S_{i}= \begin{cases}\left(\bigcup_{k=1}^{\frac{d-1}{2}}\left\{\left[a^{i d+k}, a^{-i d-k}\right]\right\}\right) \cup\left\{\left[1_{G}, a^{i d} b\right]\right\} & \text { if } i \text { is even } \\ \left(\bigcup_{k=\frac{d+1}{2}}^{d-1}\left\{\left[a^{i d+k}, a^{-i d-k}\right]\right\}\right) \cup\left\{\left[1_{G}, a^{i d} b\right]\right\} & \text { if } i \text { is odd }\end{cases}
$$

With this definition, we take $H_{i}=<a^{d}, b>$ for all these sets. As in case B, all elements of $\Omega_{2} \cup \Omega_{2}^{-1}$ are covered by the union of differences sets $\partial\left(S_{0}\right) \cup \cdots \cup \partial\left(S_{d^{\prime}-1}\right)$. The involutions not covered by these differences are now chosen to construct the other $S_{i}$ 's as $S_{i}=\left\{\left[1_{G}, a^{k} b\right]\right\}$ with $k \not \equiv 0 \bmod d$, with group $H_{i}=G$.

Propositions 10 and 11, together with the three constructions of Proposition 12, give the following result:

Theorem 13. A dihedral 1-factorization of $K_{m \times n}$ exists for all $m, n$ with $m n$ even.

Proof. Suppose $m=2^{u} d$ and $n=2^{v} d^{\prime}$ with $d$ and $d^{\prime}$ both odd. The cases $(u, v)=$ $(0,1),(0,2)$ or $(1,0)$ are all covered by Proposition 12 . For $u=0$ and $v \geq 3, u=1$ and $v \geq 1$, or $u \geq 2$ and $v=1$, Theorem 2 proves the existence of a 1 -factorization of $K_{2^{u} d \times 2^{v-1} d^{\prime}}$ with a cyclic group, and Proposition 10 can be applied to obtain the dihedral factorization of $K_{2^{u}} d \times 2^{v} d^{\prime}$.

For $u \geq 2, v=0$ or $v \geq 2$, a 1-factorization of $K_{2^{u-1} d \times 2^{v} d^{\prime}}$ with a cyclic sharply transitive automorphism group is given by Theorem 2. Applying Proposition 11, we obtain the required dihedral 1-factorization of $K_{2^{u}} d \times 2^{v} d^{\prime}$. 


\section{References}

[1] Arrigo Bonisoli and Domenico Labbate, One-factorization of complete graphs with vertex-regular automorphism groups, Journal of Combinatorial Designs 10 (2002), 116.

[2] Simona Bonvincini and Giuseppe Mazzuoccolo, Abelian one-factorizations in infinite graphs, European Journal of Combinatorics 31 (2010), 1847-1852.

[3] Marco Buratti, Abelian 1-factorizations of the complete graph, European Journal of Combinatorics 22 (2001), 291-295.

[4] Alan Hartman and Alexander Rosa, Cyclic one-factorization of the complete graph, European Journal of Combinatorics 6 (1985), 45-58.

[5] Heather Jordon and Joy Morris, Cyclic hamiltionian cycle systems of the complete graph minus a 1-factor, Discrete Mathematics 308 (2007), 2440-2449.

[6] Gàbor Korchmàros, Sharply transitive 1-factorizations of the complete graph with an invariant 1-factor, Journal of Combinatorial Designs 2 (1994), 185-196.

[7] Giuseppe Mazzuoccolo and Gloria Rinaldi, Sharply transitive 1-factorization of complete multipartite graphs, Electronic Journal of Combinatorics 17 (2010), no. R77.

[8] Gloria Rinaldi, Nilpotent 1-factorizations of the complete graph., Journal of Combinatorial Designs 13 (2005), 393-405. 